\title{
BROTAÇÃO DE GEMAS EM MACIEIRAS 'IMPERIAL GALA' E 'FUJI SUPREMA' PELO USO DE ERGER ${ }^{\circledR}$ E NITRATO DE CÁLCIO ${ }^{1}$
}

\author{
FERNANDO JOSÉ HAWERROTH², JOSÉ LUIZ PETRI ${ }^{3}$, \\ GABRIEL BERENHAUSER LEITE ${ }^{4}$, FLAVIO GILBERTO HERTER ${ }^{5}$
}

RESUMO - Apesar da alta eficiência de algumas substâncias químicas na indução da brotação de frutíferas de clima temperado, a elevada toxicidade apresentada por esses compostos é um dos principais problemas relacionados ao seu uso. Resultados preliminares indicam que Erger ${ }^{\circledR}$, composto à base de nitrogênio, combinado com nitrato de cálcio, tem efeito similar à cianamida hidrogenada, com a vantagem de ser menos agressivo ao ambiente. Assim, o presente trabalho teve por objetivo avaliar a eficiência da combinação de Erger $^{\circledR}$ e nitrato de cálcio como indutor de brotação em macieira. O experimento foi conduzido em Caçador-SC, no ano de 2007, sendo efetuada a aplicação de diferentes indutores de brotação em macieiras das cultivares 'Imperial Gala' e 'Fuji Suprema'. Os indutores de brotação testados foram Erger $^{\circledR}$ a 3\% + nitrato de cálcio a $3 \%$, Erger $^{\circledR}$ a $5 \%$ + nitrato de cálcio a $5 \%$, $\operatorname{Erger}^{\circledR}$ a $7 \%$ + nitrato de cálcio a $7 \%$, óleo mineral 3,2\% + cianamida hidrogenada $0,34 \%$ e a testemunha (sem aplicação). Foram avaliadas as datas de ocorrência das fases de início, plena e fim de floração, brotação de gemas axilares e terminais, uniformidade da brotação de gemas axilares e frutificação efetiva. Os indutores de brotação aumentaram significativamente a brotação de gemas axilares e terminais, assim como aumentaram a uniformidade da brotação e a coincidência de florescimento das cultivares Imperial Gala e Fuji Suprema. As combinações de Erger ${ }^{\circledR}$ e nitrato de cálcio apresentaram desempenho similar à cianamida hidrogenada e ao óleo mineral na brotação de gemas e na uniformização da brotação, mostrando ser uma eficiente alternativa para a indução da brotação em macieira. Em função do efeito negativo sobre a frutificação efetiva em macieiras 'Imperial Gala', indica-se a utilização de $\operatorname{Erger}^{\circledR}$ e nitrato de cálcio em concentrações inferiores a 7\%.

Termos para indexação: Malus domestica, indução da brotação, coincidência de florescimento, frutificação efetiva.

\section{BUDBREAK IN 'IMPERIAL GALA' AND 'FUJI SUPREMA' APPLES BY USING ERGER ${ }^{\circledR}$ AND CALCIUM NITRATE}

\begin{abstract}
Despite the high efficiency of some chemicals on budbreak induction of temperate fruit trees, the high toxicity showed by these compounds is one of the main problems related to its use. Preliminary results indicated that Erger ${ }^{\mathbb{R}}$, an organic nitrogen compound, combined with calcium nitrate, has similar effect to hydrogen cyanamide, with the advantage of being less dangerous to the environment. Thus, the present study aimed to evaluate the efficiency of $\operatorname{Erger}^{\circledR}$ and calcium nitrate as budbreak promoter in apple trees. The experiment was carried out in Caçador/SC, in 2007, where it was done the application of different budbreak promoters in 'Imperial Gala' and 'Fuji Suprema' apples. The budbreak promoters tested were Erger ${ }^{\circledR} 3 \%+$ calcium nitrate 3\%, Erger $^{\circledR} 5 \%+$ calcium nitrate $5 \%$, Erger ${ }^{\circledR} 7 \%+$ calcium nitrate $7 \%$, mineral oil 3.2\% + $0.34 \%$ hydrogen cyanamide and control (without application). It was evaluated occurrence dates of early, full and end blooming stages, budbreak of axillaries and terminal buds, axilliary budbreak uniformity and fruit set. The budbreak promoters increased significantly axillary and terminal budbreak, and it increased the budbreak uniformity and the blooming overlap of 'Imperial Gala' and 'Fuji Suprema' apples. Because of the negative effects on fruit set of 'Imperial Gala' apples, it is indicated $\operatorname{Erger}^{\circledR}$ and calcium nitrate application at concentrations below 7\%.
\end{abstract}

Index terms: Malus domestica, budbreak induction, blooming overlap, fruit set.

\footnotetext{
(Trabalho 152-09). Recebido em: 17-06-2009. Aceito para publicaçaõ em: 19-01-2010.

${ }^{2}$ Eng. Agr., MSc., Bolsista de Doutorado do CNPq, Programa de Pós-graduação em Agronomia - Concentração em Fruticultura de Clima Temperado, Universidade Federal de Pelotas, C.P. 354, 96010-900, Pelotas-RS. fjhawerroth@gmail.com;

${ }^{3}$ Eng. Agr., MSc., Epagri - Estação Experimental de Caçador, C.P. 591, 89500-000, Caçador-SC. petri@epagri.sc.gov.br;

${ }^{4}$ Eng. Agr., Dr., Epagri - Estação Experimental de Caçador, C.P. 591, 89500-000, Caçador-SC. gabriel@epagri.sc.gov.br;

${ }^{5}$ Eng. Agr., Dr., Universidade Federal de Pelotas, C.P. 354, 96010-900, Pelotas-RS. flavioherter@gmail.com.
} 


\section{INTRODUÇÃO}

A brotação e o florescimento na cultura da macieira (Malus domestica Borkh.) manifestam-se normalmente quando cultivada em regiões com alta acumulação de frio durante o período hibernal. Quando cultivada em regiões com insuficiente acúmulo de frio para atender a suas necessidades fisiológicas, a exemplo das condições do Sul do Brasil, a macieira exibe brotação deficiente e desuniforme (PETRI; LEITE, 2004), redução e irregularidade na abertura das gemas florais e vegetativas (EL-AGAMY et al., 2001), as quais podem reduzir o volume e a qualidade dos frutos produzidos.

Segundo Petri et al. (2006), a maior parte da cultura da macieira no Brasil está localizada em áreas onde a exigência em frio das principais cultivares utilizadas, Gala e Fuji, não é plenamente satisfeita. Nestes casos, a utilização de produtos químicos, que promovam e uniformizem a brotação e a floração, é prática comum na viabilização dos cultivos de frutíferas de clima temperado (CITADIN et al., 2006). De acordo com George et al. (2002), substâncias indutoras de brotação podem ser utilizadas para reduzir o requerimento em frio de cultivares de baixa e média exigência, permitindo seu cultivo em áreas que não proporcionam acúmulo de frio suficiente. Da mesma forma, os indutores de brotação podem ser utilizados para modular a época de brotação, floração e maturação dos frutos de espécies frutíferas temperadas, mesmo em regiões onde a dormência é superada normalmente, de modo a captar as épocas preferenciais de mercado, e para aumentar o número das gemas brotadas em espécies com forte dominância apical, aumentando sua floração de modo a restabelecer sua capacidade produtiva.

Reguladores de crescimento e compostos nutricionais podem ser utilizados na indução da brotação de frutíferas (MOHAMED, 2008), porém dentre as opções de indutores de brotação disponíveis no mercado, a cianamida hidrogenada destaca-se pela eficiência na indução da brotação em macieira, sendo utilizada juntamente com óleo mineral no sistema brasileiro de produção de maçãs (PETRI et al. 2006). Apesar da alta eficiência desta combinação, a elevada toxicidade apresentada pela cianamida hidrogenada constitui um dos principais problemas relacionados ao seu uso. Para Erez (2000), as principais características desejáveis em substâncias químicas indutoras de brotação são possuir grande eficiência na indução da brotação, baixo custo de utilização e mínima toxidade às plantas e ao ambiente. Apesar da existência de grande número de substâncias efetivas na indução da brotação, poucas são aceitas e utilizadas comercialmente, sendo o alto custo de utilização e a elevada toxicidade dos compostos os principais fatores restritivos.

A necessidade de restringir cada vez mais o uso de substâncias sintéticas na condução dos pomares, preconizada pelos programas de Produção Integrada de Frutas, torna a questão da superação da dormência pelo uso de agentes químicos em plantas frutíferas um fator limitante para a atividade no Brasil (SANHUEZA et al., 2003). Frente à necessidade de se dispor de produtos com menor toxicidade e agressão ao meio ambiente, o desenvolvimento de novos compostos que possuam tais características, aliadas à eficiência na indução da brotação, é almejado (HAWERROTH, 2009). Seguindo esta tendência, trabalhos preliminares têm apontado a combinação de Erger $^{\circledR}$, composto à base de nitrogênio e nitrato de cálcio como eficiente indutor da brotação de gemas em macieira (PETRI, 2005; PETRI et al., 2008b). Neste sentido, o presente trabalho teve por objetivo avaliar a eficiência da combinação de $\operatorname{Erger}^{\circledR}$ e de nitrato de cálcio como indutores de brotação em macieira.

\section{MATERIAL E MÉTODOS}

O estudo foi realizado no município de Caçador-SC (latitude $26^{\circ} 46^{\prime} \mathrm{S}$, longitude $51^{\circ} \mathrm{W}$, altitude 960 metros), durante o ano de 2007, em pomar pertencente à Epagri/Estação Experimental de Caçador. Foram utilizadas macieiras das cultivares Imperial Gala e Fuji Suprema com 12 anos de idade, enxertadas sobre o porta-enxerto M.26, efetuando-se a aplicação de diferentes indutores de brotação sobre as mesmas.

O pomar utilizado apresentava densidade de plantio de 2.500 plantas ha- ${ }^{-1}$, com espaçamento de 4 $\mathrm{m}$ entre linhas e $1 \mathrm{~m}$ entre plantas, sendo as plantas conduzidas no sistema em líder central e manejadas de acordo com recomendações do sistema de produção da macieira (SANHUEZA et al., 2006). O esquema de polinização adotado baseava-se na utilização de duas cultivares produtoras, sendo Imperial Gala polinizadora da cultivar Fuji Suprema, e vice-versa.

O delineamento experimental utilizado foi em blocos casualizados, seguindo um arranjo fatorial (5x2) obtido da combinação de cinco níveis do fator indutor de brotação e dois níveis do fator cultivar. Foram utilizadas seis repetições, onde cada repetição foi composta por uma planta. Os níveis estudados para o fator indutor de brotação foram: 1) testemunha (sem aplicação); 2) Erger a 3\% + nitrato de cálcio a $3 \%$; 3) Erger a $5 \%$ + nitrato de cálcio a 5\%;4) 
Erger a 7\% + nitrato de cálcio a 7\%; 5) óleo mineral a $3,2 \%$ + cianamida hidrogenada a $0,34 \%$. Como fonte de cianamida hidrogenada e óleo mineral, foram utilizados os produtos comercias Dormex ${ }^{\circledR}(49 \%$ de ingrediente ativo) e Triona ${ }^{\circledR}(80 \%$ de ingrediente ativo), respectivamente.

A aplicação dos indutores de brotação foi realizada quando já haviam sido acumuladas 876,5 unidades de frio, segundo modelo Carolina do Norte modificado (EBERT et al., 1986), estando as gemas entre os estádios A e B, segundo escala fenológica ilustrada em Iuchi (2006). A aplicação dos indutores de brotação foi realizada com pulverizador costal motorizado, tendo-se aplicado volume médio de calda de $1.000 \mathrm{~L} \mathrm{ha}^{-1}$.

A partir do início da brotação das gemas, foram realizadas avaliações fenológicas para determinar as datas de ocorrência das fases de início, plena e final de floração para cada tratamento. $\mathrm{O}$ início de floração foi considerado quando as plantas apresentavam $5 \%$ de flores abertas, a plena floração quando verificado mais de $80 \%$ de flores abertas e o fim de floração foi dado quando as últimas flores estavam abertas. A partir dos dados fenológicos obtidos, calculou-se o intervalo, em dias, compreendido entre a aplicação dos indutores de brotação e a data de início da floração, juntamente com a duração dos períodos de floração, os quais foram utilizados para verificar a coincidência de floração existente entre as cultivares estudadas dentro de cada nível do fator indutor de brotação.

A brotação de gemas axilares e terminais foi avaliada aos 27 dias após a aplicação dos tratamentos. A brotação de gemas axilares foi estimada por meio da contagem de gemas brotadas e não brotadas em cinco brindilas previamente selecionadas, localizadas no terço médio da planta. A partir das observações destas cinco brindilas, foram obtidos os valores médios de brotação de gemas axilares e os desviopadrão respectivos a cada tratamento, calculando-se os coeficientes de variação dentro de cada tratamento estudado, os quais foram denominados índices de heterogeneidade da brotação de gemas axilares. A porcentagem de gemas terminais brotadas foi estimada a partir de uma ramificação lateral selecionada em cada planta. A frutificação efetiva foi obtida da relação entre o número de cachos florais contados durante a plena floração e o número de frutos ([número de frutos/cachos florais]x100), sendo as contagens realizadas na mesma ramificação lateral utilizada na estimativa da brotação de gemas terminais.

As variáveis expressas em percentagem foram transformadas pela equação $(x+1)^{1 / 2}$, a fim de atender às pressuposições da análise de variância. Os resultados foram submetidos à análise de variância, e as variáveis que revelaram significância a $5 \%$ de probabilidade de erro, tiveram as médias comparadas pelo teste de Tukey, a 5\% de probabilidade de erro.

\section{RESULTADOS E DISCUSSÃO}

Na Figura 1, observa-se que a duração do florescimento diferiu entre as cultivares e níveis do fator indutor de brotação estudados. Quando não efetuada a aplicação de indutor de brotação, as macieiras 'Imperial Gala' apresentaram período de florescimento de 16 dias, enquanto, nas macieiras 'Fuji Suprema', nesta mesma condição, este foi de apenas 9 dias. Segundo Petri e Leite (2004), o prolongamento das fases de brotação e de floração na cultura da macieira é sintoma típico de insuficiência em frio hibernal, onde o aumento do período de florescimento pode dificultar a realização de algumas práticas culturais, como raleio e controle de doenças, devido à ocorrência de diferentes estádios fenológicos dentro de uma mesma planta. Na cultivar Imperial Gala, a aplicação dos indutores de brotação reduziu significativamente o período de florescimento em relação às testemunhas, contribuindo para a uniformização das práticas culturais subsequentes, enquanto na cultivar Fuji Suprema o período de florescimento manteve-se em 9 dias nos demais tratamentos, embora o início da floração tenha ocorrido antecipadamente com o uso dos indutores de brotação. Avaliando os indutores de brotação separadamente em cada cultivar, não foram observadas diferenças significativas entre as aplicações de Erger $^{\circledR}$ e nitrato de cálcio com aplicações de óleo mineral e cianamida hidrogenada quanto à duração da floração.

Quanto à época de florescimento, o efeito dos indutores de brotação manifestou-se igualmente nas duas cultivares em estudo, justificado pela não ocorrência de interação significativa entre os fatores indutor de brotação e cultivar (Tabela 1). O uso dos indutores de brotação reduziu significativamente o intervalo compreendido entre a aplicação dos tratamentos e o início de floração, antecipando a floração em 5,5 dias em relação às plantas em que não foi realizado tratamento para a indução da brotação. Em condições de inverno ameno, onde as exigências em frio não são completamente satisfeitas, cultivares com distintos requerimentos em frio apresentam grande variabilidade na época de florescimento, de um ano para outro (PETRI et al. 2008a). A assincronia de florescimento entre cultivares é claramente observada no tratamento-testemunha (Figura 1), onde o pleno florescimento da cultivar Imperial Gala coincide com o término da floração da cultivar Fuji 
Suprema. Os diferentes tratamentos de Erger $^{\circledR}$ e nitrato de cálcio, assim como o óleo mineral associado à cianamida hidrogenada aumentaram a sincronia do florescimento entre as cultivares Imperial Gala e Fuji Suprema, evidenciado pela maior coincidência nas datas de plena floração entre estas cultivares. A ocorrência de florescimento simultâneo de cultivares produtoras e suas polinizadoras, com coincidência da plena floração entre cultivares, aumenta a possibilidade de ocorrer altos índices de frutificação efetiva (SOLTÉSZ, 2003). A resposta diferenciada entre os níveis do fator indutor de brotação quanto à época de florescimento evidencia a importância do uso de indutores de brotação como estratégia de manejo para maximizar a polinização através da maior sincronização da floração entre cultivares de importância comercial e suas polinizadoras (HAWERROTH, 2009).

Os indutores de brotação utilizados proporcionaram aumento significativo da brotação de gemas axilares em relação ao tratamento-testemunha, tanto na cultivar Imperial Gala quanto na cultivar Fuji Suprema (Tabela 2). No tratamento-testemunha, a brotação de gemas axilares foi significativamente superior na cultivar Fuji Suprema, apresentando $30,3 \%$ das gemas axilares brotadas, enquanto a cultivar Imperial Gala apresentava apenas 12,8\% de brotação. Com a aplicação dos indutores de brotação, os percentuais de brotação de gemas axilares mostraram-se superiores a 52,1\% e $61,5 \%$ nas cultivares Imperial Gala e Fuji Suprema, respectivamente. Não foram observadas diferenças entre as combinações de Erger $^{\circledR}$ e nitrato de cálcio avaliadas quanto à brotação de gemas axilares, sendo os resultados similares ao observado no tratamento com cianamida hidrogenada e óleo mineral, corroborando os resultados obtidos por Petri et al. (2008b). Estes autores observaram que a aplicação de Erger $^{\text {R }}$ e nitrato de cálcio exibiu resultados semelhantes e, em algumas situações, superiores aos tratamentos com cianamida hidrogenada e óleo mineral quanto à brotação de gemas axilares.

A interação entre os fatores cultivar e indutor de brotação não foi significativa quanto a heterogeneidade da brotação de gemas axilares, demonstrando que a resposta frente ao uso dos indutores de brotação para esta variável foi a mesma para ambas as cultivares (Tabela 2). No tratamento-testemunha, na média das cultivares em estudo, os índices de heterogeneidade da brotação de gemas axilares foi de elevada magnitude, apresentando-se superior a $100 \%$, indicando elevada desuniformidade da brotação entre ramos de uma mesma planta. Quando efetuada a aplicação dos indutores de brotação, a heterogeneidade da brotação de gemas axilares foi reduzida para índices inferiores a $41,2 \%$, evidenciando efeito positivo destes na uniformização da brotação, assim como relatado por Erez (2000) e Petri et al. (2006). Não foram verificadas diferenças significativas na heterogeneidade da brotação de gemas axilares entre as combinações de $\operatorname{Erger}^{\circledR}$ com nitrato de cálcio em relação ao tratamento com cianamida hidrogenada e óleo mineral.

No tratamento-testemunha, as macieiras 'Imperial Gala' e 'Fuji Suprema' apresentaram reduzida brotação de gemas terminais, exibindo, em média, 19,2\% das gemas terminais brotadas (Tabela 3). O uso de indutores de brotação proporcionou aumento significativo da brotação de gemas terminais em relação ao tratamento-testemunha, sendo esta resposta observada em ambas as cultivares estudadas. Independentemente da concentração de Erger $^{\circledR}$ e nitrato de cálcio utilizada, a resposta na brotação de gemas terminais foi similar à observada no tratamento com óleo mineral e cianamida hidrogenada, cuja brotação de gemas terminais se apresentou superior a 75,7\%.

Em relação à frutificação efetiva, as cultivares estudadas responderam diferentemente aos tratamentos com indutores de brotação (Figura 2). Na cultivar Fuji Suprema, não houve diferença significativa entre os níveis do fator indutor de brotação em relação à frutificação efetiva. Em contrapartida, verificou-se drástica redução da frutificação efetiva nas macieiras da cultivar 'Imperial Gala', sendo o tratamento com Erger $^{\circledR}$ a $7 \%$ + nitrato de cálcio a $7 \%$ o que proporcionou maior redução da frutificação efetiva, apresentando índices próximos a 7,8\%. Petri (2005) observou que a aplicação de Erger $^{\circledR}$ a $7 \%$ + nitrato de cálcio a $10 \%$ reduziu a frutificação efetiva, determinando significativa diminuição da produção de frutos em macieiras 'Gala', podendo ser justificado pelo rápido desenvolvimento foliar competindo com a abundante floração e desenvolvimento dos frutos observados neste tratamento, e devido à concentração da floração que propicia um curto período para a polinização e atividade de insetos polinizadores. Segundo Erez (2000) e Petri et al. (2006), esta mesma resposta pode ser obtida quando utilizadas altas concentrações de cianamida hidrogenada e óleo mineral nos tratamentos para indução da brotação na cultura da macieira. $\mathrm{Na}$ cultivar 'Imperial Gala', os tratamentos com Erger $^{\circledR}$ a 3\% + nitrato de cálcio a 3\% e Erger ${ }^{\circledR}$ a $5 \%$ + nitrato de cálcio a $5 \%$ proporcionaram índices de frutificação efetiva similares ao observado nas macieiras tratadas com óleo mineral a $4 \%$ e cianamida hidrogenada a $0,34 \%$. 


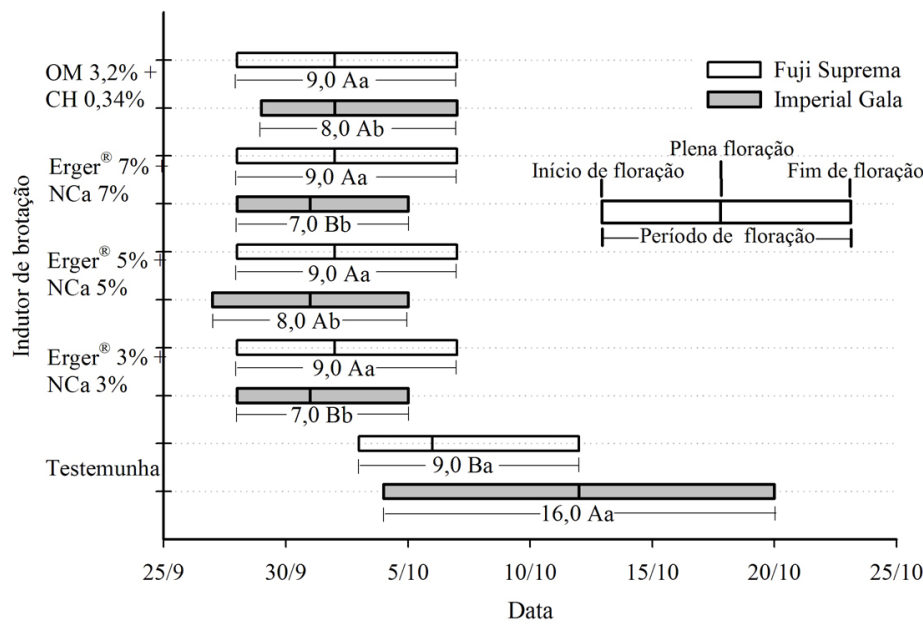

$\mathrm{F}$ (indutor de brotação $\mathrm{x}$ cultivar) $=216,09 * *$

** Valor de $\mathrm{F}$ significativo a $1 \%$ de probabilidade;

$\mathrm{NCa}=$ nitrato de cálcio; $\quad \mathrm{OM}=$ óleo mineral; $\quad \mathrm{CH}=$ cianamida hidrogenada;

Médias seguidas de mesma letra minúscula dentro de cultivar, e médias seguidas de mesma letra maiúscula dentro de indutor de brotação, não diferem significativamente pelo teste Tukey a 5\% de probabilidade de erro.

FIGURA 1 - Duração do período de florescimento em macieiras 'Imperial Gala' e 'Fuji Suprema' tratadas com diferentes indutores de brotação. Caçador-SC, 2007.

TABELA 1 - Intervalo entre aplicação dos tratamentos e início da floração de macieiras 'Imperial Gala' e 'Fuji Suprema' tratadas com diferentes indutores de brotação. Caçador-SC, 2007.

\begin{tabular}{lccc}
\hline \multirow{2}{*}{ Indutor de brotação } & \multicolumn{3}{c}{ Intervalo entre aplicação dos tratamentos e início da floração } \\
\cline { 2 - 4 } & Imperial Gala & Fuji Suprema & Média \\
\hline \multirow{2}{*}{ Testemunha } & 29,2 & 27,8 \\
Erger $^{\circledR} 3 \%+\mathrm{NCa} 3 \%$ & 23,0 & 23,0 & $28,5 \mathrm{a}$ \\
Erger $^{\circledR} 5 \%+\mathrm{NCa} 5 \%$ & 22,0 & 23,0 & $23,0 \mathrm{~b}$ \\
Erger $^{\circledR} 7 \%+\mathrm{NCa} 7 \%$ & 23,0 & 23,0 & $22,5 \mathrm{~b}$ \\
OM 3,2\%+CH 0,34\% & 24,0 & 23,2 & $23,0 \mathrm{~b}$ \\
\hline Média Geral & $24,2 \mathrm{~A}$ & $24,0 \mathrm{~A}$ \\
$\mathrm{CV}(\%)=5,31$ & \multicolumn{3}{c}{ F (indutor de brotação x cultivar) $=0,01 \mathrm{~ns}$} \\
\hline
\end{tabular}

ns - interação entre os fatores indutor de brotação e cultivar não significativa, pelo teste $\mathrm{F}$, a $5 \%$ de probabilidade de erro. Médias seguidas da mesma letra maiúscula na linha, e médias seguidas da mesma letra minúscula na coluna, não diferem estatisticamente, pelo teste de Tukey, a $5 \%$ de probabilidade de erro. 
TABELA 2 - Brotação de gemas axilares e heterogeneidade da brotação de gemas axilares em macieiras ‘Imperial Gala'e 'Fuji Suprema' tratadas com diferentes indutores de brotação. Caçador-SC, 2007.

Gemas axilares

\begin{tabular}{|c|c|c|c|c|c|}
\hline \multirow[t]{2}{*}{ Indutor de brotação } & \multicolumn{2}{|c|}{ Brotação } & \multicolumn{3}{|c|}{ Heterogeneidade } \\
\hline & $\begin{array}{c}\text { Imperial } \\
\text { Gala } \\
\end{array}$ & $\begin{array}{c}\text { Fuji Supre- } \\
\text { ma }\end{array}$ & $\begin{array}{c}\text { Imperial } \\
\text { Gala } \\
\end{array}$ & $\begin{array}{c}\text { Fuji Su- } \\
\text { prema }\end{array}$ & Média \\
\hline & \multicolumn{5}{|c|}{.$\%}$. \\
\hline Testemunha & $12,8 \mathrm{Bb}$ & $30,3 \mathrm{Ab}$ & 111,0 & 90,2 & $100,5 \mathrm{a}$ \\
\hline Erger $^{\circledR} 3 \%+\mathrm{NCa} 3 \%$ & $52,1 \mathrm{Aa}$ & $61,5 \mathrm{Aa}$ & 44,9 & 37,5 & $41,2 b$ \\
\hline Erger $^{\circledR} 5 \%+\mathrm{NCa} 5 \%$ & $67,4 \mathrm{Aa}$ & $62,7 \mathrm{Aa}$ & 32,5 & 26,9 & $29,7 b$ \\
\hline Erger $^{\circledR} 7 \%+\mathrm{NCa} 7 \%$ & $70,9 \mathrm{Aa}$ & $68,5 \mathrm{Aa}$ & 34,8 & 25,3 & $30,1 b$ \\
\hline $\mathrm{OM} 3,2 \%+\mathrm{CH} 0,34 \%$ & $68,0 \mathrm{Aa}$ & 77,9Аа & 36,4 & 19,6 & $28,0 \mathrm{~b}$ \\
\hline Média & 54,3 & 60,2 & $51,9 \mathrm{~A}$ & $39,9 \mathrm{~A}$ & 45,9 \\
\hline $\mathrm{F}$ (cultivar $\mathrm{x}$ indutor de brotação) & \multicolumn{2}{|c|}{$2,88^{*}$} & \multicolumn{3}{|c|}{$0,17 \mathrm{~ns}$} \\
\hline CV $(\%)$ & \multicolumn{2}{|c|}{10,89} & \multicolumn{3}{|c|}{30,64} \\
\hline
\end{tabular}

ns - interação entre os fatores cultivar e indutor de brotação não significativa, pelo teste $\mathrm{F}$, a $5 \%$ de probabilidade de erro; * interação entre os fatores cultivar e indutor de brotação significativa, pelo teste F, a 5\% de probabilidade de erro; NCa - nitrato de cálcio; OM - óleo mineral; $\mathrm{CH}$ - cianamida hidrogenada; Médias seguidas da mesma letra maiúscula na linha, e médias seguidas da mesma letra minúscula na coluna não diferem estatisticamente, pelo teste de Tukey, a 5\% de probabilidade de erro.

TABELA 3 - Brotação de gemas terminais de macieiras 'Imperial Gala' e 'Fuji Suprema', tratadas com diferentes indutores de brotação. Caçador-SC, 2007.

\begin{tabular}{|c|c|c|c|}
\hline \multirow{2}{*}{ Indutor de brotação } & \multicolumn{3}{|c|}{ Brotação de gemas terminais } \\
\hline & Imperial Gala & Fuji Suprema & Média \\
\hline & \multicolumn{3}{|c|}{..$\%$} \\
\hline Testemunha & 18,5 & 19,8 & $19,2 \mathrm{~b}$ \\
\hline $\operatorname{Erger}^{\circledR} 3 \%+\mathrm{NCa} 3 \%$ & 83,7 & 68,8 & $76,3 \mathrm{a}$ \\
\hline Erger $^{\circledR} 5 \%+\mathrm{NCa} 5 \%$ & 88,2 & 79,9 & $83,6 \mathrm{a}$ \\
\hline Erger $^{\circledR} 7 \%+\mathrm{NCa} 7 \%$ & 93,3 & 58,0 & $75,7 \mathrm{a}$ \\
\hline $\mathrm{OM} 3,2 \%+\mathrm{CH} 0,34 \%$ & 87,6 & 65,5 & $76,5 \mathrm{a}$ \\
\hline Média & $74,3 \mathrm{~A}$ & $58,2 \mathrm{~B}$ & 66,2 \\
\hline $\mathrm{F}$ (cultivar $\mathrm{x}$ indutor de brotação) & \multicolumn{3}{|c|}{$0,92 \mathrm{~ns}$} \\
\hline CV $(\%)$ & \multicolumn{3}{|c|}{16,10} \\
\hline
\end{tabular}

ns - interação não significativa, pelo teste $\mathrm{F}$, a 5\% de probabilidade de erro; $\mathrm{NCa}$ - nitrato de cálcio; $\mathrm{OM}$ - óleo mineral; $\mathrm{CH}$ - cianamida hidrogenada; Médias seguidas da mesma letra maiúscula na linha, e médias seguidas da mesma letra minúscula na coluna não diferem estatisticamente, pelo teste de Tukey, a 5\% de probabilidade de erro. 


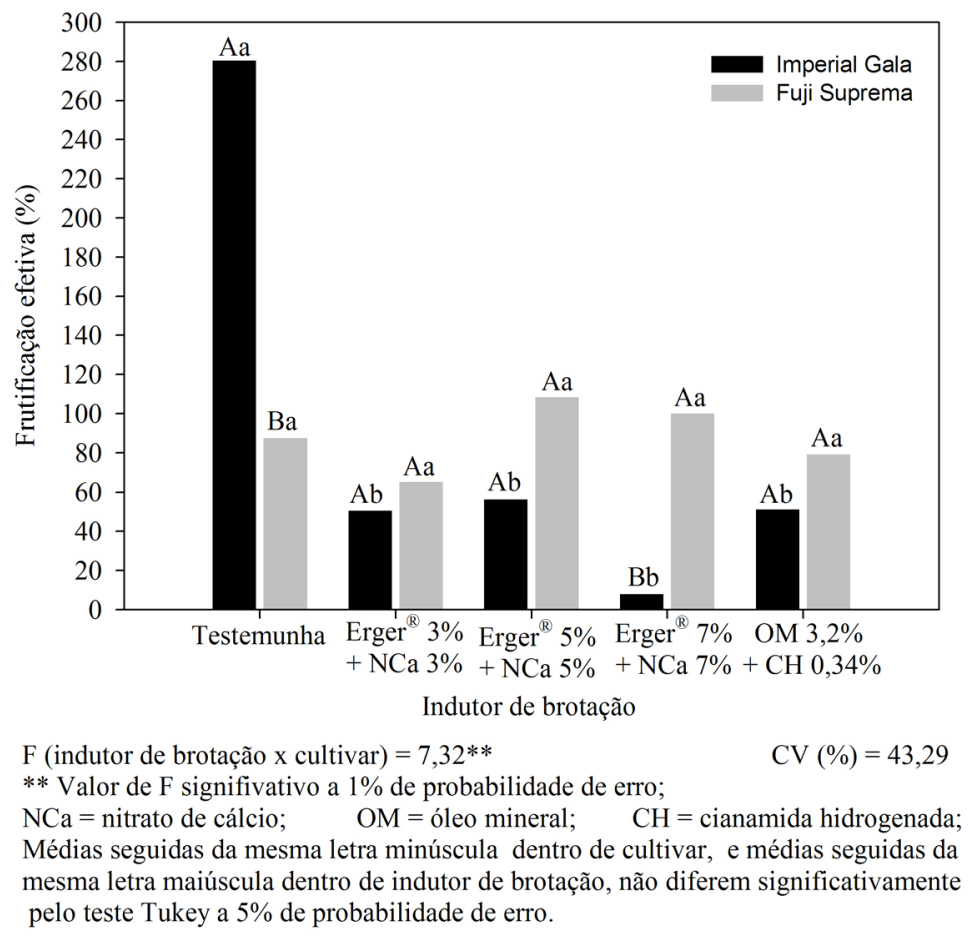

FIGURA 2 - Frutificação efetiva em macieiras 'Imperial Gala' e 'Fuji Suprema' tratadas com diferentes indutores de brotação. Caçador-SC, 2007.

\section{CONCLUSÕES}

Os indutores de brotação aumentam significativamente a brotação de gemas axilares e terminais, assim como aumentam a uniformidade da brotação e a coincidência de florescimento das cultivares Imperial Gala e Fuji Suprema. As combinações de Erger $^{\circledR}$ e nitrato de cálcio apresentam desempenho similar à cianamida hidrogenada e ao óleo mineral na brotação de gemas e na uniformização da brotação, mostrando ser uma eficiente alternativa para indução da brotação em macieira. Indica-se a utilização de $\operatorname{Erger}^{\circledR} \mathrm{e}$ nitrato de cálcio em concentrações inferiores a 7\%, pois acima deste limite pode ocorrer diminuição da frutificação efetiva em macieiras 'Imperial Gala' pelo uso de tais produtos.

\section{REFERÊNCIAS}

CITADIN, I.; BASSANI, M.H.; DANNER, M.A.; MAZARO, S.M.; GOUVÊA, A. Uso de cianamida hidrogenada e óleo mineral na floração, brotação e produção do pessegueiro 'Chiripá'. Revista Brasileira de Fruticultura, Jaboticabal, v.28, n.1, p.32-35, 2006.

EBERT, A.; PETRI, J.L.; BENDER, R.J.; BRAGA, H.J. First experiences with chill units models is southern Brazil. Acta Horticulturae, The Hague, v.184, p.89-96, 1986.

EL-AGAMY, S.Z.; MOHAMED, A.K.A.; MOSTAFA, F.M.A.; ABDALLAH, A.Y. Effect of GA3, hydrogen cyanamide and decapitation on budbreak and flowering of two apple cultivars under the warm climate of southern Egypt. Acta Horticulturae, The Hague, v.565, p.109-114, 2001.

EREZ, A. Bud dormancy: phenomenon, problems and solutions in the tropics and subtropics. In: EREZ, A. Temperate fruit crops in warm climates. Boston: Kluwer Academic Publishers, 2000. p.17-48. 
GEORGE, A.P.; BROADLEY, R.H.; NISSEN, R.J.; WARD, G. Effects of new rest-breaking chemicals on flowering, shoot production and yield of subtropical tree crops. Acta Horticulturae, The Hague, v.575, p.835-840, 2002.

HAWERROTH, F.J. Dormência de gemas sob influência da temperatura durante o período hibernal e resposta produtiva da macieira pelo uso de indutores de brotação. 2009. 123 f. Dissertação (Mestrado em Agronomia - Fruticultura de Clima Temperado) - Faculdade de Agronomia Eliseu Maciel, Universidade Federal de Pelotas, Pelotas, 2009.

IUCHI, V.L. Botânica e fisiologia. In: EPAGRI. A cultura da macieira. Florianópolis: Epagri, 2006. p. 59-104.

MOHAMED, A.K.A. The effect of chilling, defoliation and hydrogen cyanamide on dormancy release, bud break and fruiting of Anna apple cultivar. Scientia Horticulturae, Amsterdam, v.118, p.25-32, 2008.

PETRI, J.L. Alternativas para quebra de dormência em fruteiras de clima temperado. In: ENCONTRO NACIONAL SOBRE FRUTICULTURA DE CLIMA TEMPERADO, 8., 2005, Fraiburgo. Anais... Caçador: Epagri, 2005. v.1, p.125-133.

PETRI, J.L.; HAWERROTH, F.J.; LEITE, G.B. Fenologia de espécies silvestres de macieira como polinizadora das cultivares Gala e Fuji. Revista Brasileira de Fruticultura, Jaboticabal, v.30, n.4, p.868-874, 2008a.
PETRI, J.L.; LEITE, G.B. Consequences of insufficient winter chilling on apple tree bud-break. Acta Horticulturae, The Hague, v.662, p.53-60, 2004.

PETRI, J.L.; LEITE, G.B.; PUTTI, G.L. Apple tree budbreak promoters in mild winter conditions. Acta Horticulturae, The Hague, v.774, p.291-296, 2008b.

PETRI, J.L.; PALlADINI, L.A.; POLA, A.C. Dormência e indução à brotação em macieira. In: EPAGRI. A cultura da macieira. Florianópolis, 2006. p.261-297.

SANHUEZA, R.M.V.; ANDRIGUETO, J.R.; KOSOSKI, A.R. Situação atual da produção integrada de frutas no Brasil. In: MELO, G.W.B.; SEBBEN, S.S (Ed.). SEMINÁRIO BRASILEIRO DE PRODUÇÃO INTEGRADA DE FRUTAS, 5., 2003, Bento Gonçalves. Anais... Bento Gonçalves: EmbrapaCNPUV, 2003. p.23-25.

SANHUEZA, R.M.V.; PROTAS, J.F.S.; FREIRE, J.M. Manejo da macieira no sistema de produção integrada de frutas. Bento Gonçalves: Embrapa Uva e Vinho, 2006. 164p.

SOLTÉSZ, M. Apple. In: KOZNA, P.; NYÉKI, J.; SOLTÉSZ, M.; SZABO, Z. Floral biology, pollination and fertilisation zone fruit species and grape. Budapest: Akadémia Kiadó, 2003. p.237-316. 